\title{
Smartphone-Based Self-Testing of COVID-19 Using Breathing Sounds
}

\author{
Miad Faezipour, PhD, ${ }^{1,2, *}$ and Abdelshakour Abuzneid, $P h D^{1, *}$ \\ Departments of ${ }^{1}$ Computer Science \& Engineering and \\ ${ }^{2}$ Biomedical Engineering, University of Bridgeport, Bridgeport, \\ Connecticut, USA. \\ *These authors contributed equally to this work
}

\begin{abstract}
Telemedicine could be a key to control the world-wide disruptive and spreading novel coronavirus disease (COVID19) pandemic. The COVID-19 virus directly targets the lungs, leading to pneumonia-like symptoms and shortness of breath with life-threatening consequences. Despite the fact that self-quarantine and social distancing are indispensable during the pandemic, the procedure for testing COVID-19 contraction is conventionally available through nasal swabs, saliva test kits, and blood work at healthcare settings. Therefore, devising personalized self-testing kits for COVID-19 virus and other similar viruses is heavily admired. Many e-health initiatives have been made possible by the advent of smartphones with embedded software, hardware, high-performance computing, and connectivity capabilities. A careful review of breathing sounds and their implications in identifying breathing complications suggests that the breathing sounds of COVID-19 contracted users may reveal certain acoustic signal patterns, which is worth investigating. To this end, acquiring respiratory data solely from breathing sounds fed to the smartphone's microphone strikes as a very appealing resolution. The acquired breathing sounds can be analyzed using advanced signal processing and analysis in tandem with new deep/ machine learning and pattern recognition techniques to separate the breathing phases, estimate the lung volume, oxygenation, and to further classify the breathing data input into healthy or unhealthy cases. The ideas presented have the potential to be deployed as self-test breathing monitoring apps for the ongoing global COVID-19 pandemic, where users can check their breathing sound pattern frequently through the app.
\end{abstract}

Keywords: e-health, telemedicine, home health monitoring, technology, sensor technology
A lthough the novel coronavirus disease (COVID-19) has disrupted the whole world, telemedicine may be the immediate target to control the spreading pandemic. Many e-health initiatives have been made possible by the advent of smartphones. The embedded software and hardware have permitted numerous smartphonebased health care monitoring systems to emerge. Physiological data are collected using the smartphone's embedded sensors (i.e., camera, microphone, text, clicks, and fingerprints) or external hardware and wearable sensors attached to the smartphone. There are already $>100,000$ smartphone-based health care-related applications (apps) available and the number is expected to grow exponentially. ${ }^{1,2}$ The acquired data are analyzed using the respective app to provide adequate responses, or uploaded to the cloud for further analysis and actuation. Consequently, health care professionals would be informed to solicit diagnosis and prognosis.

The COVID-19 pandemic already resulted in $>330,000$ deaths across the globe. It is primarily attributed to the fact that the human lungs are targeted by the virus and breathing is afflicted. $^{3-5}$ Projections reveal that there would be a second phase of COVID-19 and possibly other pandemics approaching. Although self-quarantine and social distancing are indispensable during the pandemic, the procedure for testing COVID-19 contraction is conventionally available through nasal swabs, saliva test kits, and blood work at health care settings. Therefore, devising personalized self-testing kits for COVID-19 virus and other similar viruses is heavily favored. Such self-tests will serve as an early step before medical doctors order further procedures such as lung CT and X-Ray. In addition, the pandemic has affected millions of people in a short span of time, causing much burden on hospitals and medical professionals. Providing accurate, real-time, and inexpensive testing will facilitate speedy prediagnosis to a large population, which could drastically help control the spread of the pandemic.

Many pulmonary-related diseases and breathing disorders (asthma, pneumonia, lung cancer, etc.) are a direct result of changes in the airway tract structure and cause symptoms such as airway inflammation, airflow limitation, shortness of breath, coughs, and crackle/wheezing sounds when breathing. ${ }^{6,7}$ The COVID-19 virus directly targets the lungs, leading to pneumonia-like symptoms and shortness of breath with life-threatening consequences. Breathing disorders are 
traditionally diagnosed using tests such as pulmonary function testing, chest high-resolution computed tomography, and bronchoscopies at clinics. ${ }^{8}$ Whereas personalized and automated respiratory monitoring systems offer decision support for prevention, early detection, and treatment planning. Smartphone apps are a valuable commodity that could be tailored as respiratory monitoring systems for pandemic control.

To collect respiratory data, doctors use digital stethoscopes to listen to the lung's acoustic signals and spirometers to measure lung volume and capacity by gauging the airflow in the lung. Moreover, doctors perform tests to check the blood oxygenation flowing from the lungs. Photoplethysmographic (PPG) biometric signatures that deal with volumetric changes in the blood flow through infrared light absorption can quantify this measure. Contact and/or noninvasive PPG pulse signals acquired from a pulse oximeter are clinically used to measure blood oxygenation. Furthermore, the ComputerRespiratory-Sound-Analysis society ${ }^{9}$ has defined a standard of $>160$ terms pertaining to respiratory sounds for diagnosing

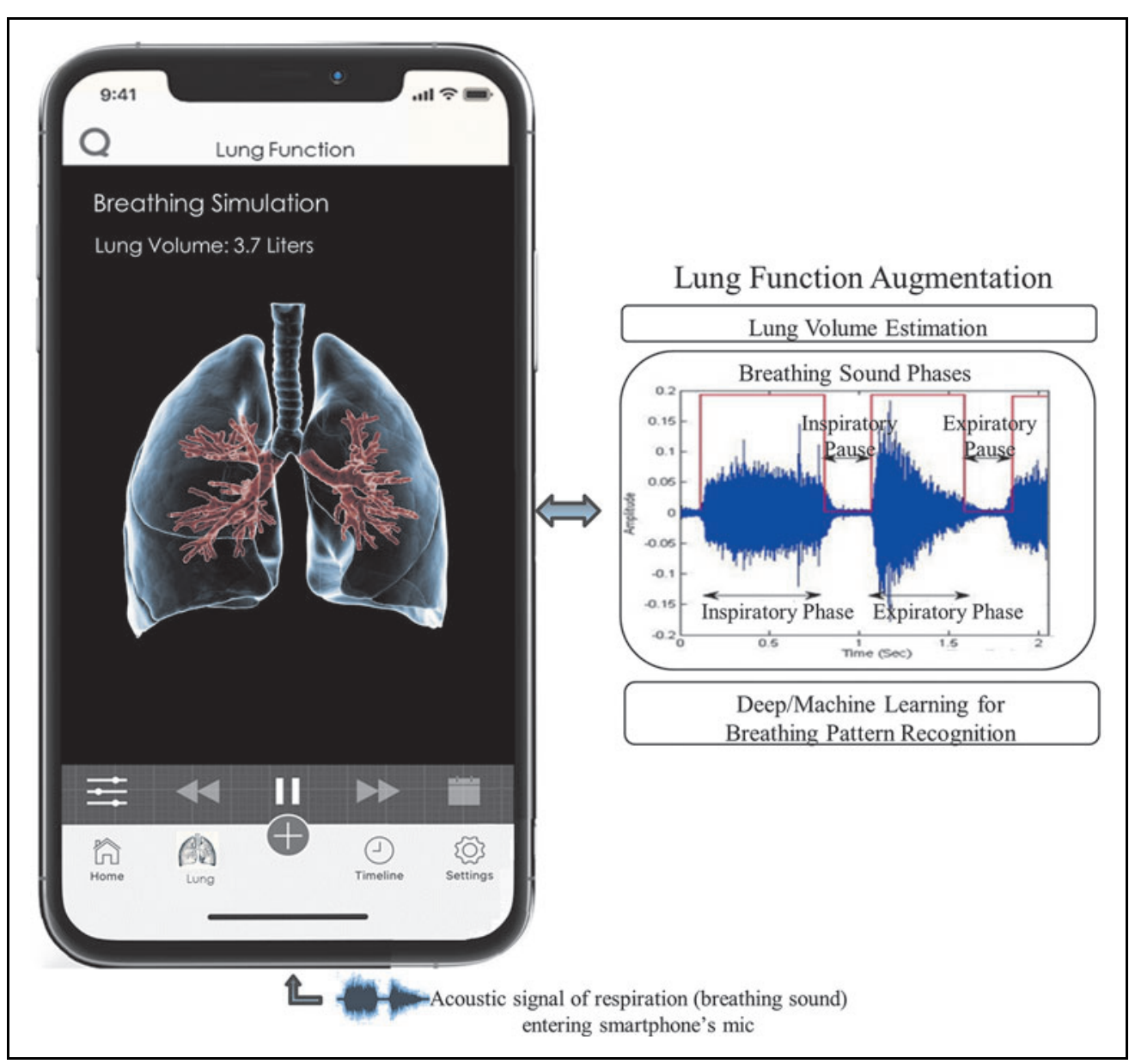

Fig. 1. Lung function augmentation with breathing sounds. various breathing conditions. Among those include the generic terms of normal breath sounds, adventitious sounds, and breath sounds (which include both normal and adventitious sounds). Figure 1 depicts four breathing phases fetched from the breathing sound (acoustic signal of respiration). The inspiratory phase refers to the inhale/inflow of air to the lungs, whereas the inspiratory pause is when the incoming airflow stops. The expiratory phase denotes the exhale/outflow of air from the lungs, whereas the expiratory pause refers to when the outgoing airflow stops. Crackles and wheezes are the main adventitious sounds that are exemplified by certain frequency characteristics in the inspiratory phases, whereas cough and snoring sounds have certain frequency responses in the inhale and exhale phases. Rhonchus, squawk, and stridor are among other adventitious sounds with certain frequency responses in the inspiration phase. Most adventitious sounds are associated with one or more lung/breathing complications. ${ }^{10}$ Several frequency and wavelet-based approaches have been applied to identify the breathing phases and adventitious sounds. ${ }^{10,11}$

In addition, the lung volume and oxygenation can be modeled and approximated with a good accuracy using breathing sounds by considering the energy of the acoustic signal of respiration in each phase of airflow to/from the lungs. ${ }^{12-14}$

A careful review of various adventitious sounds suggests that COVID-19 breathing sounds may also have certain time/frequency components, which is worth investigating. A team of researchers from the University of Cambridge and Carnegie Melon University are currently working on a collaborative effort to create a voice database (including voice of the user speaking, breathing, and coughing) for COVID-19. ${ }^{15}$ However, detecting COVID-19 solely from breathing sounds has not been studied as of yet. Thus, this research proposes a novel insight in this regard. To accomplish this, a database of breathing sounds should be generated that includes statistically significant samples of the coronavirus contracted 


\section{FAEZIPOUR AND ABUZNEID}

breathing sounds in addition to breathing sounds of healthy control subjects. To be accurate, the database of breathing sounds should include many other breathing disorder-related sounds as well as to perform a multiclass classification task.

Moreover, shortness of breath (which occurs for most COVID-19 contracted cases) is often due to insufficient oxygenation that also appears as lower lung volume (capacity). As blood oxygenation also drops in coronavirus infected patients, this measure reveals important information regarding COVID-19. Thus, for better accuracy and reliability, we believe lung volume data of sample users obtained by the spirometer as well as blood oxygenation measures collected from pulse oximeter devices should also be included in the generated data set, and associated with each breathing sound recording. The collected data samples should be annotated/labeled (healthy or unhealthy: COVID-19 infected) by clinical staff and medical specialists to set the ground truth. Deep and machine learning techniques can then be used to learn from training the database samples and distinguish what category a new breathing sound input belongs to.

Acquiring respiratory data solely from breathing sounds fed to the smartphone's microphone strikes as a very appealing resolution. The idea here is to devise an interactive smartphonebased Breathing Simulation App for self-diagnosis of breathing complications from breathing sounds. The app additionally offers lung function augmentation by displaying high-definition virtual lung animations inflating and deflating as the user inhales and exhales in real time (Fig. 1). Thus, we further suggest using only breathing sound patterns to model the lung volume and oxygenation as well. The acquired breathing sounds can be analyzed using Mel-frequency analysis in tandem with new deep/machine learning and pattern recognition techniques (i.e., recurrent neural networks) to separate the breathing phases, and estimate the lung volume as well as oxygenation. Once the database is trained, our artificial intelligence (AI) algorithms would be able to correlate these data with the breathing sounds through regression models of the annotated samples to provide an estimate of the lung volume and oxygenation from only breathing sounds. The AI classification models could further classify the breathing data input into healthy or unhealthy cases (i.e., corresponding to a breathing disease or complication such as pneumonia or COVID-19).

The breathing app for self-testing of COVID-19 would especially be most beneficial for those with higher risks of severe illness (e.g., older adults or patients with serious underlying medical conditions) as well as clinical cohorts of high-risk exposure patients, where quick frequent daily self-testing can be performed. Individuals exposed to the public (such as transit workers and police/force/emergency medical services (EMS) workers) and cultural settings with risk for infection (e.g., multifamily settings with multiple house members working with high risk for COVID-19 exposure settings), in addition to those in meat packing plants or the front-line grocery store workers, are especially in the high-risk exposure category. This additionally emphasizes the need for developing testing models of the breathing app from the breathing sound database. To date, there is no complete, annotated, labeled, open-source, and publicly available database of breathing sounds that researchers, physicians, medical staff, scientists, and digital signal processing (DSP) engineers can refer to for identifying COVID-19. Through this opinion article, we are suggesting that such comprehensive database be established so the machine learning and AI analysis ideas could be applied to design the breathing simulation app and automate the detection process. Baseline testing of the high-risk individuals over time would reveal changes in breathing sound patterns measured temporally, further leading to strong data analytics with greater predictability.

The presented ideas suggest that breathing sounds solely collected from smartphones may reveal the onset of certain breathing complications such as that in COVID-19. The lifesaving impacts would be enormous if the ideas are fully translated to personalized smartphone-based breathing sound simulation apps for preliminary self-testing of COVID-19.

\section{Authors' Contributions}

Drs. Faezipour and Abuzneid both contributed to the development of the content of this article. Both authors are heavily involved in the Digital/Biomedical Embedded Systems \& Technology (D-BEST) laboratory research, located at the School of Engineering, University of Bridgeport, CT, USA.

\section{Acknowledgment}

The authors thank Dr. Marcia Prenguber, ND, FABNO, who is the director of Naturopathic Medicine at the University of Bridgeport (UB) Clinics and UB Health Sciences Center, for her fruitful discussions and for providing great insight on the ideas of this article from a medical/clinical perspective.

\section{Disclosure Statement}

No competing financial interests exist.

\section{Funding Information}

No funding was received for this article.

\section{REFERENCES}

1. Gupta G. Are Medical Apps the future of medicine? Med J Armed Forces India 2013;69:105-106. 
2. Bradway $M$, Carrion $C$, Vallespin $B$, Saadatfard O, Puigdomènech $E_{\text {, }}$ Espallargues M, Kotzeva A. mHealth assessment: Conceptualization of a global framework. JMIR mHealth uHealth 2017;5:e60.

3. World Health Organization. Available at https://www.who.int/ (last accessed May 22, 2020).

4. Johns Hopkins Coronavirus Resource Center. Available at https:// coronavirus.jhu.edu (last accessed May 22, 2020).

5. IHME COVID-19 Projections. Available at https://covid19.healthdata.org/ projections (last accessed May 22, 2020).

6. Centers for Disease Control and Prevention, National Center for Health Statistics. Available at www.cdc.gov/nchs (last accessed May 22, 2020)

7. Lung Cancer Fact Sheet, American Lung Association. Available at www.lung.org (last accessed May 22, 2020).

8. Brown MS, Goldin JG, Suh RD, McNitt-Gray MF, Sayre JW, Aberle DR. Lung micronodules: Automated method for detection at thin-section CT-initial experience. Radiology 2003;226:256-262.

9. Sovijärvi A, Vanderschoot J, Earis J. Standardization of computerized respiratory sound analysis. Crit Care Med 1997;156:974-987.

10. Reichert $S$, Gass R, Brandt C, Andrès E. Analysis of respiratory sounds: State of the art. Clin Med Circ Respirat Pulm Med 2008;2:45-58.

11. Abushakra A, Faezipour M. Acoustic signal classification of breathing movements to virtually aid breath regulation. IEEE J Biomed Health Inform 2013;17:493-500.

12. Abushakra A, Faezipour M. Estimating lung capacity from acoustic signal of respiration. IEEE EMBS Conference on Micro and Nanotechnology in Medicine. IEEE, Ka'anapali, HI, USA (IEEE MNM'12). 2012;19.
13. Abushakra A, Faezipour M. Lung capacity estimation through acoustic signal of breath. IEEE 12th International Conference on Bioinformatics \& Bioengineering (IEEE BIBE'12) 2012;386-391.

14. Abushakra A, Faezipour M. Augmenting breath regulation using a mobile driven virtual reality therapy framework. IEEE J Biomed Health Inform 2014;18:746-752.

15. Researchers struggle with COVID-19 voice detection apps-Report. Available at https://pharmaphorum.com/news/researchers-struggle-with-covid-voicedetection-apps-report/ (last accessed May 22, 2020).

Address correspondence to: Miad Faezipour, PhD

Department of Computer Science \& Engineering

School of Engineering

221 University Avenue University of Bridgeport

Bridgeport, CT 06604-7620

USA

E-mail:mfaezipo@bridgeport.edu

Received: April 19, 2020

Revised: May 22, 2020

Accepted: May 26, 2020

Online Publication Date: June 2, 2020 